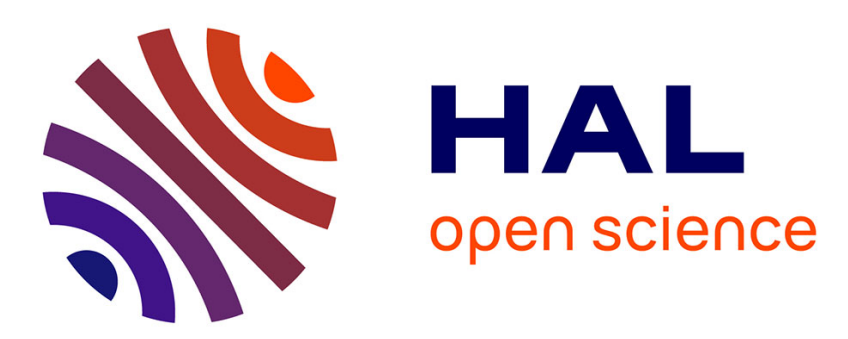

\title{
Un cadre d'analyse multidimensionnel de débats en ligne asynchrones
}

\author{
Yves Kuster, Geneviève Lameul
}

\section{To cite this version:}

Yves Kuster, Geneviève Lameul. Un cadre d'analyse multidimensionnel de débats en ligne asynchrones. EPAL - Echanger Pour Apprendre en Ligne, Jun 2009, Grenoble, France. hal-02011987

\section{HAL Id: hal-02011987 \\ https://hal.science/hal-02011987}

Submitted on 8 Feb 2019

HAL is a multi-disciplinary open access archive for the deposit and dissemination of scientific research documents, whether they are published or not. The documents may come from teaching and research institutions in France or abroad, or from public or private research centers.
L'archive ouverte pluridisciplinaire HAL, est destinée au dépôt et à la diffusion de documents scientifiques de niveau recherche, publiés ou non, émanant des établissements d'enseignement et de recherche français ou étrangers, des laboratoires publics ou privés. 


\title{
UN CADRE D’ANALYSE MULTIDIMENSIONNEL DE DEBATS EN LIGNE ASYNCHRONES
}

\author{
Yves Kuster, Geneviève Lameul \\ CREAD - UBO-IUFM de Bretagne - Rennes 2
}

Résumé : Les forums de discussion sont des modalités de formation de plus en plus répandues dans les dispositifs utilisant les TIC. Si leur mise en place ne pose aujourd'hui plus de problème d'un point de vue technique, il n'en va pas de même en ce qui concerne l'analyse des contenus qu'ils produisent. Nous nous attachons dans ce chapitre à présenter le cadre d'analyse que nous nous sommes construits au fil du temps pour approcher au plus près la dynamique et la construction de connaissances qui s'opèrent dans cette modalité de formation. Nous nous appuyons sur un cas pratique pour l'expliciter : l'analyse d'un forum-débat (54 messages postés par 33 participants sur une durée de deux mois) que nous analysons depuis plusieurs années nous permet d'apporter quelques illustrations au fil de notre présentation. Ce chapitre se veut faire un point plus spécifiquement méthodologique dans la perspective que des formateurs ou intervenants dans des forums puissent également s'en servir comme outils d'intervention.

Mots clés : forum-débat, analyse de pratiques, compétences professionnelles, approche méthodologique intégrée 
La formation professionnelle initiale des professeurs stagiaires de sciences de la vie et de la Terre (SVT) à l'institut universitaire de formation des maîtres (IUFM) de Bretagne est une formation professionnelle par alternance au sein de laquelle l'analyse de la pratique professionnelle occupe une place importante. Elle est centrée sur un dispositif de formation hybride qui articule des temps de formation en présentiel (séances en petits groupes, visites de classe) et des temps de formation à distance. Les formateurs SVT réalisent en particulier, à distance et de manière asynchrone, des forums de discussion électroniques tutorés que nous avons baptisés forums débats. Il s'agit, sur une période de temps donnée, d'environ un mois, d'amener les professeurs stagiaires à débattre sur des questions professionnelles polémiques.

Nous approfondissons depuis plusieurs années (2005-08) l'analyse de ce type de débats en ligne asynchrones dans l'objectif d'en comprendre l'impact sur la construction de compétences par les professeurs stagiaires y participant. On peut penser que dans ce type de débat, s'expriment et s'entrecroisent des propos en rapport avec divers types de compétences : des compétences professionnelles au regard du référentiel métier mis en place récemment par le ministère de l'éducation nationale ${ }^{1}$ et des compétences de type conversationnel en particulier autour de l'argumentation. De manière complémentaire, il nous a paru intéressant de nous pencher sur les modes sociaux de co-construction de connaissances au sein du forum débat. Ainsi les questions auxquelles nous tentons de porter une réponse se structurent autour de trois dimensions d'analyse :

- Analyse de la dimension épistémique ou comment détecter des indices fiables de construction de compétences professionnelles au sein des propos tenus?

- Analyse de la dimension argumentative ou comment se structure l'argumentation dans ce type de forum de discussion? Comment dégager les grandes lignes de la conceptualisation par l'argumentation qui se construit au fil des échanges?

- Analyse de la dimension sociale ou par quel processus socio-collaboratif s'opère la construction de savoir identifié ? Quels mécanismes font fructifier les interactions entre les professeurs stagiaires?

De cette triple interrogation, nous avons construit au fil du temps un cadre d'analyse multidimensionnel de nos forums débats qui s'inspire du cadre d'analyse de Weinberger et Fischer (2006). Cette communication décrit cette approche originale d'un forum de discussion selon trois dimensions complémentaires : dimension épistémique, dimension argumentative et dimension sociale. L'exemple utilisé comme fil conducteur de notre présentation est un forum débat, proposé durant l'année scolaire 2004-2005 à des professeurs stagiaires de SVT et qui a pour thème : l'interrogation écrite surprise (IES), pratique professionnelle que les professeurs stagiaires rencontrent couramment dans les établissements où ils effectuent leur stage et qu'ils ont connue en tant qu'élèves. Ce type de débat se situe en aval de journées de formation en présentiel consacrées au thème général de l'évaluation des élèves. Il se présente comme une forme d'écriture professionnelle permettant la mobilisation de la pensée des professeurs stagiaires sur des questions professionnelles délimitées. Les formateurs visent une certaine forme de conceptualisation des pratiques échangées sur le thème abordé et la coconstruction de stratégies d'enseignement permettant éventuellement de dépasser les contradictions initialement exprimées.

\footnotetext{
${ }^{1}$ Cahier des charges des IUFM (Bulletin officiel $n^{\circ} 1$ du 04 janvier 2007)
} 


\section{Le cadre théorique d'analyse}

Notre cadre théorique s'inspire de celui de Weinberger et Fischer (2006) qui développent un modèle d'analyse multidimensionnel de la co-construction de connaissances par l'argumentation dans un environnement $\mathrm{CSCL}^{2}$.

\subsection{Dimension épistémique}

Le pari de la formation professionnelle initiale en IUFM est de tenter une mise en synergie entre savoir théorique et savoir pragmatique au bénéfice de la construction de compétences professionnelles. Dès que l'on parle de compétences, la difficulté consiste à s'accorder sur une définition du concept. Pour des auteurs comme Le Boterf (1994), Perrenoud (1997), Beckers (2002), Allal (2002) la compétence est un «savoir mobiliser dans l'action» des ressources internes (cognitives) et externes afin de traiter une tâche ou afin de résoudre un problème complexe. Tous ces auteurs nous renvoient finalement au constructivisme piagétien, aux notions de schèmes d'action et de classes de situations dont Vergnaud (1990) affinera les concepts dans sa théorie des champs conceptuels. C'est dans ce contexte que notre choix s'est porté sur le modèle de Coulet (2005) qui considère la construction d'une compétence comme la mise en oeuvre d'une activité productive de schèmes d'action qui s'accompagne de formes de régulation sur certains éléments constitutifs de ces schèmes (régulation en boucle courte sur les règles d'action du schème ou en boucle longue sur les invariants opératoires du schème) (figure 1).

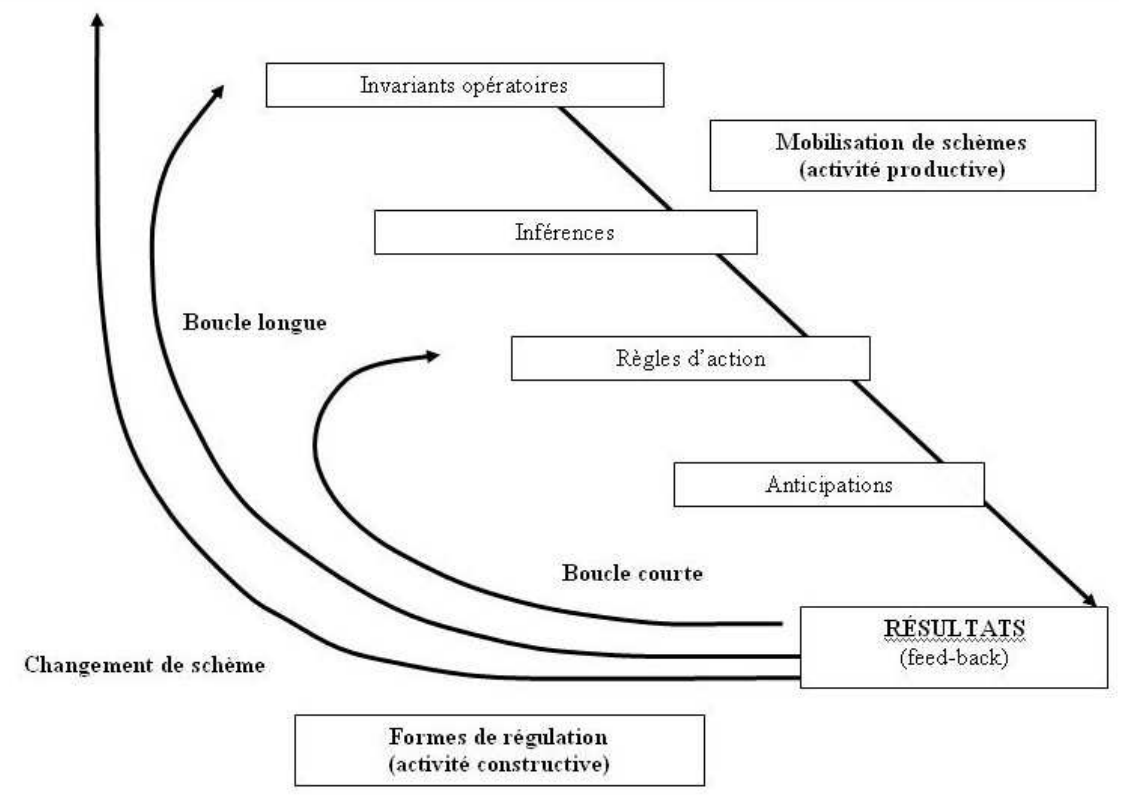

Figure 1 : Une modélisation de la construction d'une compétence (Coulet, 2005)

Nous postulons que les messages postés par les professeurs stagiaires dans un forum débat prennent le statut de «fenêtres » ouvertes à la fois sur leurs conceptions du métier et sur leurs pratiques effectives. Les propos exprimés par les professeurs stagiaires dans le débat IES peuvent s'interpréter comme une forme de verbalisation de schèmes d'action produits, mis en œuvre, interrogés ou tout simplement en gestation en rapport avec des situations de classe vécues ou rapportées autour de l'IES.

\footnotetext{
${ }^{2}$ CSCL : Computer Supported Collaborativ Learning
} 


\subsection{Dimension argumentative}

Il est important de bien définir la nature des pratiques argumentatives dans ce type de forum de discussion. Nous pensons qu'un forum débat est favorable à la mise en place de séquences argumentatives (Kuhn 1991). Dans une séquence argumentative les arguments se construisent en premier pour justifier une ou plusieurs positions. Ensuite, les partenaires d'apprentissage construisent des contre arguments pour défier et reconsidérer ces positions. Les contre arguments facilitent les activités méta-cognitives, incitant l'apprenant à revoir l'argument initial (Leitao, 2000). Enfin les apprenants peuvent construire ce qu'on appelle des répliques qui contribuent en finale à affiner les positions initiales. Selon Leitao, les étapes individuelles d'une séquence argumentative représentent un cycle de construction de connaissances. C'est en pesant arguments et contre arguments pour essayer de résoudre des problèmes complexes que les participants d'un débat peuvent acquérir de nouvelles connaissances sur le domaine considéré mais aussi acquérir la capacité à envisager un problème selon des perspectives multiples.

La recherche de séquences argumentatives au sein du forum débat IES nous a permis d'en révéler la trame argumentative et d'en dégager les potentialités en terme d'acquisition de connaissances $\left(\mathrm{cf} 3^{\circ}\right)$. En revanche, cela ne nous dit rien sur la structure des arguments ou contre arguments développés. La structuration de l'argumentation dans un forum débat peut être étudiée en se référant au modèle de Toulmin (1958/1993) qui identifie six composantes dans la construction d'un argument (figure2).

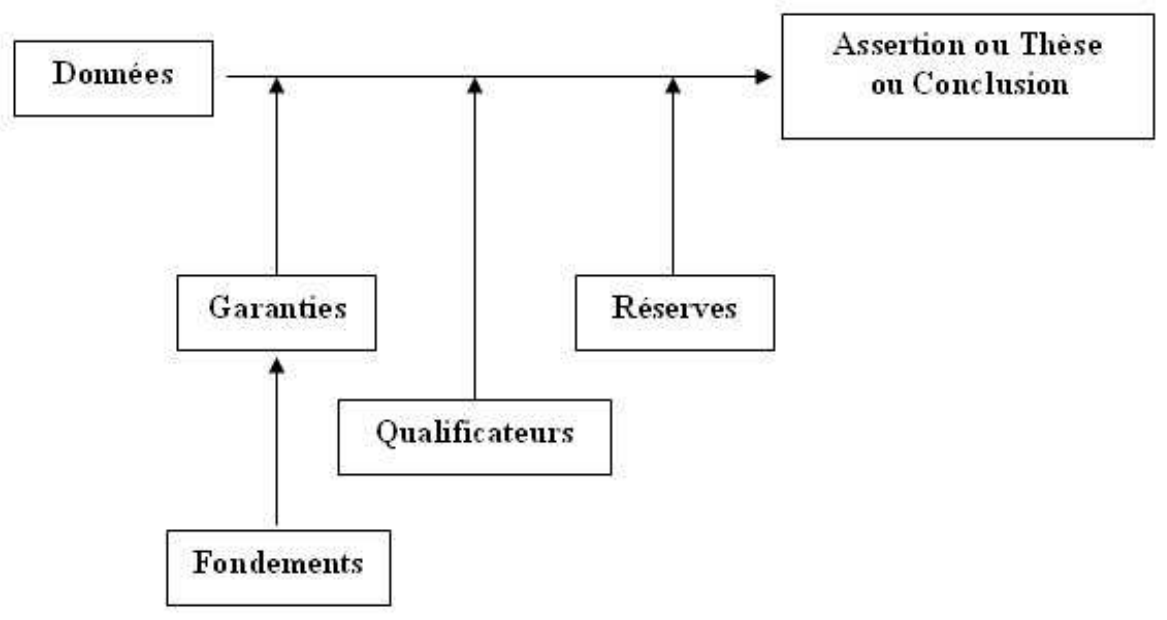

Figure 2 : Le modèle de Toulmin

Au constat que ce modèle est difficile à appliquer dans sa globalité pour un débat sur le mode de la conversation (en ligne ou non) (Desjardins, 2002), nous nous sommes tournés vers les travaux de Weinberger et Fischer (2006) qui utilisent un modèle plus simple, inspiré du modèle de Toulmin. Ces deux chercheurs proposent d'étudier la structuration des arguments d'un débat électronique asynchrone selon 4 catégories: l'assertion simple, l'assertion qualifiée, l'assertion garantie, l'assertion garantie et qualifiée.

Les assertions sont des déclarations qui mettent en évidence la position que les professeurs stagiaires prennent. Les garanties sont des données (observations, témoignages, expériences, références théoriques...) qui présentent les raisons pour lesquelles une assertion peut être considérée comme valide. Des liens logiques signalent souvent la manière dont une assertion est reliée aux données la garantissant. Enfin, les qualificateurs sont des données qui limitent la validité d'une assertion à un public ou des circonstances spécifiques. 


\subsection{Dimension sociale}

La manière dont les participants à ces forums débats construisent des arguments peut être distribuée entre plusieurs membres d'un groupe et, d'après Weinberger et Fischer (2006), selon cinq degrés d'investissement du point de vue social bien différent :

- l'externalisation, caractérisée par les situations où les professeurs stagiaires apportent leur contribution sans faire référence aux autres contributions dans le forum (Webb 1989)

- l'élicitation, correspondant aux situations où l'un des participants au forum utilise ses partenaires comme ressources en leur posant des questions (Dillenbourg et al, 1996).

- la construction d'un consensus rapide, se traduisant par le fait qu'un professeur stagiaire accepte les propositions d'un ou de plusieurs autres stagiaires, soit parce qu'il est convaincu, soit parce qu'il désire que le débat avance même sans être convaincu.

- la construction d'un consensus par intégration, caractérisée par des situations où les professeurs stagiaires intègrent à leur propos, les arguments d'autres professeurs stagiaires

- la construction d'un consensus basé sur le conflit qui aboutit à un consensus en reprenant les différentes alternatives et en tentant de les dépasser

Cependant, nous savons bien que la collaboration ne produit pas systématiquement les effets d'apprentissage et de socialisation escomptés (Johnson et Johnson, 1999). Pour tenter de comprendre si les traces de savoir collectif produisent un effet au niveau individuel, la question des interactions sociales et des prises de postures diversifiées au sein des échanges (Lameul, 2008) s'est imposée au fil de notre travail. L'analyse épistémique tente de repérer des schèmes ou des composantes de schèmes que chaque intervenant dans le forum évoque, soit au regard de ses propres pratiques, soit au regard de celles des autres intervenants. A ce niveau, il s'agit bien de schèmes individuels mais que les interactions verbales dans le cadre $\mathrm{du}$ forum vont d'une part, pouvoir éventuellement affecter, et d'autre part, permettre de partager. Le schème est alors appréhendé dans ses caractéristiques culturelles : on peut repérer une organisation de l'activité assez largement partagée par plusieurs individus ${ }^{3}$. Nous constatons, par ailleurs, des caractéristiques de ces schèmes ou composantes de schèmes plus ou moins partagées par une communauté de stagiaires. Toutefois, c'est dans l'analyse des influences des interactions sur la déconstruction / reconstruction de schèmes individuels qu'on pourrait voir véritablement quelle est la contribution du forum au développement des compétences individuelles et en quoi ces compétences individuelles tendent à converger (culture commune) ou à diverger (identité propre). (Lameul \& Kuster, 2009)

\section{La méthodologie d'analyse}

La littérature sur les forums de discussion montre essentiellement deux approches méthodologiques, la première de nature quantitative propose des analyses statistiques du contenu du forum, la seconde de nature qualitative expose une analyse didactique ou linguistique par exemple des discours postés par les intervenants. L'analyse quantitative présente comme avantage d'être dans une certaine mesure réplicable et transférable à des situations proches. Mais il s'agit le plus souvent d'une certaine forme de lecture du contenu plus qu'une interprétation réelle de ce qui est écrit. La catégorisation réalisée et quantifiée fait parfois oublier le contenu même de ce qui est écrit. L'analyse qualitative est une analyse qui entre dans le détail de ce qui est exprimé (parfois mot à mot). On accède à une compréhension

\footnotetext{
${ }^{3}$ On peut sans doute penser qu'on n'est pas très loin du concept d'habitus chez Bourdieu ou de genre chez Clot
} 
très approfondie de la situation analysée mais l'interprétation peut être très subjective, généralement non réplicable, peu transférable à d'autres situations.

Notre méthodologie d'analyse est conçue comme une approche intégrée (Chi, 1997) qui associe analyse quantitative et analyse qualitative du contenu d'un forum débat, chacune des deux analyses devant enrichir l'autre. Nous sommes partis de l'hypothèse que ce qui a trait aux trois dimensions de notre analyse est repérable par des actes de discours spécifiques et catégorisables et que la fréquence de ces actes de discours est un indicateur fiable et objectif de nos interprétations.

\subsection{Catégorisation des actes du discours à partir des modèles théoriques retenus}

Il s'agit pour chaque dimension de notre analyse de s'appuyer sur les modèles théoriques retenus et d'en extraire une grille de catégories que l'on pourra appliqué à l'ensemble des messages du forum. Les tableaux 1 et 2 donnent des exemples de grilles de catégorisation pour les dimensions épistémique et argumentative.

\begin{tabular}{|c|c|c|}
\hline \multicolumn{2}{|c|}{ Catégories } & Description \\
\hline \multirow{5}{*}{$\begin{array}{l}\text { Activité } \\
\text { productive: } \\
\text { schème d'action }\end{array}$} & Invariants opératoires & $\begin{array}{l}\text { Théorèmes en actes : ce que le sujet tient pour vrai } \\
\text { Concepts en actes : ce que le sujet tient pour pertinent }\end{array}$ \\
\hline & Inférences & $\begin{array}{l}\text { Prise d'informations, raisonnements, calculs permettant } \\
\text { l'adaptation du schème aux spécificités de la situation }\end{array}$ \\
\hline & Règles d'action & $\begin{array}{l}\text { Transformations matérielles ou symboliques que nécessite la } \\
\text { tâche pour atteindre les buts ou sous-buts qui y sont attachés }\end{array}$ \\
\hline & Anticipations & $\begin{array}{l}\text { Attentes et prédictions que le sujet se forge en termes de } \\
\text { résultats }\end{array}$ \\
\hline & Résultats & $\begin{array}{l}\text { Résultats de l'application du schème d'action à la situation. La } \\
\text { non-conformité des résultats obtenus au regard des anticipations } \\
\text { élaborées peut se traduire par des feed-back négatifs } \\
\text { (régulations) }\end{array}$ \\
\hline \multirow{3}{*}{$\begin{array}{l}\text { Activité } \\
\text { constructive: } \\
\text { régulations }\end{array}$} & $\begin{array}{l}\text { Boucle de régulation } \\
\text { courte }\end{array}$ & Modification portant sur les règles d'action du schème \\
\hline & $\begin{array}{l}\text { Boucle de régulation } \\
\text { longue }\end{array}$ & Modification portant sur les invariants opératoires du schème \\
\hline & Changement de schème & $\begin{array}{l}\text { Décentration, réorganisation de l'activité en opérant un } \\
\text { changement de schème }\end{array}$ \\
\hline \multicolumn{2}{|c|}{ Interactions non épistémiques } & Digressions sans relation avec l'objet du débat \\
\hline
\end{tabular}

Tableau 1 : Catégorisation à partir du modèle de construction de compétence (Coulet, 2005)

\begin{tabular}{|l|l|}
\hline \multicolumn{1}{|c|}{ Catégories } & \multicolumn{1}{c|}{ Description } \\
\hline Assertion simple & $\begin{array}{l}\text { Déclaration d'une opinion sans délimitation de sa validité ni apport de } \\
\text { garanties }\end{array}$ \\
\hline Assertion qualifiée & $\begin{array}{l}\text { Assertion sans apport de garanties mais comportant des éléments } \\
\text { délimitant son domaine de validité }\end{array}$ \\
\hline Assertion garantie & $\begin{array}{l}\text { Assertion sans délimitation de sa validité mais comportant l'apport de } \\
\text { garanties qui la justifient }\end{array}$ \\
\hline Assertion garantie et qualifiée & $\begin{array}{l}\text { Assertion comportant l'apport de garanties qui la justifient et la } \\
\text { délimitation de sa validité }\end{array}$ \\
\hline En dehors de la tâche & Questions, digressions ou tout énoncé sans rapport avec le débat \\
\hline
\end{tabular}

Tableau 2 : Catégorisation portant sur la structure de l'argumentation 


\subsection{Analyse quantitative du corpus de données}

\subsubsection{Segmentation du forum}

Les unités propres de notre corpus (fils, messages, phrases etc.) ne constituent pas «naturellement» des grains d'analyse adaptés. Il est nécessaire de recourir à une segmentation du discours selon un ensemble de règles qui ont été établies ici à partir des catégorisations retenues pour les trois dimensions travaillées. Le découpage relève alors du fonctionnement mental des locuteurs (découpage sémantique) plutôt que d'un découpage syntaxique. Le grain d'analyse est alors variable d'une dimension à une autre. Il peut aussi varier au sein même d'une dimension d'analyse selon les différents niveaux de connaissance recherchés dans le discours des professeurs stagiaires (Chi 1997).

Un segment ou unité d'analyse étant variable dans sa longueur, comment être objectif dans ce travail de découpage ? La solution retenue est le recours à la «méthode des juges », c'est à dire que le découpage du corpus est réalisé indépendamment par deux personnes utilisant les mêmes règles de segmentation. Le calcul d'un Kappa de Cohen (1960) nous permet d'en vérifier le taux de fiabilité. Ce test statistique permet de mesurer la qualité de l'accord réel entre des jugements qualitatifs appariés. Il exprime une différence relative entre la proportion d'accord observée $\boldsymbol{P o}$ et la proportion d'accord aléatoire $\boldsymbol{P e}$ qui est la valeur espérée sous l'hypothèse nulle d'indépendance des jugements, divisée par la quantité disponible au-delà de l'accord aléatoire.

En définitive, $K$ est un pourcentage de l'accord maximum corrigé de ce qu'il serait sous le simple effet du hasard. Dans le cas d'une étude d'accord entre deux observateurs statistiquement indépendants ayant $r$ modalités de jugement, avec $r \geq 2$, le coefficient Kappa s'écrit :

$$
K=\frac{P_{o}-P_{e}}{1-P_{e}}
$$

avec $\boldsymbol{P o}$ la proportion d'accord observée et $\boldsymbol{P e}$ la proportion d'accord aléatoire ou concordance attendue sous l'hypothèse nulle d'indépendance des jugements.

Si le niveau d'accord est insuffisant, les codeurs discutent à partir de cas de désaccord, précisent les règles de segmentation et refont le travail séparément jusqu'à obtenir un Kappa proche de 0,8 jugé satisfaisant d'après l'échelle de Landis et Koch (tableau 3)

\begin{tabular}{||l|l||}
\hline \multicolumn{1}{|c|}{ Accord } & \multicolumn{1}{c|}{ Kappa } \\
\hline Excellent & $\geq 0,81$ \\
\hline Bon & $0,80-0,61$ \\
\hline Modéré & $0,60-0,41$ \\
\hline Médiocre & $0,40-0,21$ \\
\hline Mauvais & $0,20-0,0$ \\
\hline Très mauvais & $<0,0$ \\
\hline
\end{tabular}

Tableau 3 : Degré d'accord et valeur de Kappa

\subsubsection{Codage des segments}

Le codage des segments se fait en utilisant une table de règles de codage qui reprend et détaille la grille de catégorisation retenue. A titre d'exemple, le tableau 4 propose un extrait de la table des règles concernant la dimension argumentative utilisée pour le codage du forum débat IES. On y constate que la colonne qui décrit chaque catégorie est davantage détaillée que précédemment et que des exemples de segments types sont proposés pour chaque catégorie. 
Develotte C., Mangenot F., Nissen E. (2009, coord.) Actes du colloque Epal 2009 (Echanger pour apprendre en ligne : conception, instrumentation, interactions, multimodalité), université Stendhal - Grenoble 3, 5-7 juin 2009.

\begin{tabular}{|c|c|c|}
\hline Catégories & Description & Exemples \\
\hline Assertion simple & $\begin{array}{l}\text { Déclaration d'une opinion sans délimitation de sa validité ni apport } \\
\text { de garanties }\end{array}$ & $\begin{array}{l}\text { "Le problème majeur, à mon avis, cest les rapports qu'll (l'IES) instaure entre } \\
\text { le prof qui sanctionne par le devoir quand bon lui semble et les élèves. } \\
\text { "Je pense que c'est la manière dont le devair sera amené qui donnera tel ou tel } \\
\text { climat de classe" }\end{array}$ \\
\hline $\begin{array}{l}\text { Assertion } \\
\text { qualifié }\end{array}$ & $\begin{array}{l}\text { Assertion sans apport de garanties mais comportant des éléments } \\
\text { délimitant sa validité : } \\
\text { o dans le temps (regarder la conjugaison); } \\
\text { par rapport à un groupe d'individus précis, par exemple des } \\
\text { élèves d'une classe (celle du professeur stagiaire le plus souvent) } \\
\text { ou d'un niveau (collège, lycée); } \\
\text { comme un cas particulier ou avec un certain degré de } \\
\text { généralisation (regarder les mots comme : courant, rarement, } \\
\text { généralement, probablement etc...) }\end{array}$ & $\begin{array}{l}\text { " Je pensais auparonant que les interrogations surprises représentatent presque } \\
\text { un abus d'autorité de la part des profs \$ } \\
\text { "Pour mes leve cétait bien différent. Ils lint pris je pense plus pour une } \\
\text { sanction .et avec un peu de recul je les comprend. }\end{array}$ \\
\hline $\begin{array}{l}\text { Assertion } \\
\text { garantie }\end{array}$ & $\begin{array}{l}\text { Assertion sans délimitation de sa validité mais comportant l'apport } \\
\text { de garanties qui la justifient } \\
\text { o un témoignage de situation de classe vécue en tant que } \\
\text { professeur ou en tant qu'élève, } \\
\text { - la référence à un autre professeur stagiaire ou à un expert } \\
\text { (formateur, ou conseiller pédagogique par exemples) } \\
\text { - la référence à des textes institutionnels (programmes, BO, } \\
\text { règlement intérieur...) } \\
\text { - la référence à des éléments statistiques; } \\
\text { - la référence à des concepts ou théories des sciences de } \\
\text { l'éducation }\end{array}$ & 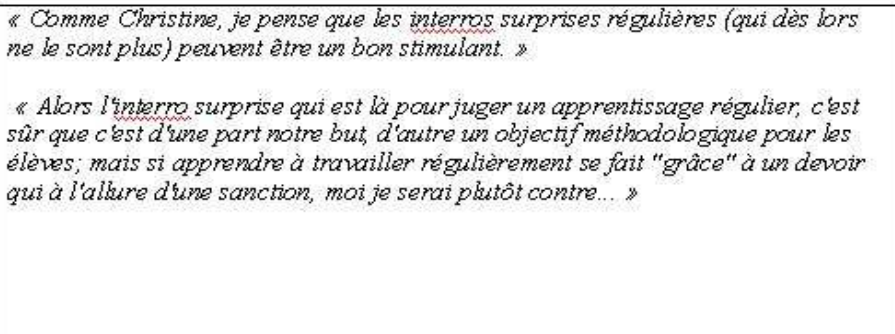 \\
\hline $\begin{array}{l}\text { Assertion } \\
\text { garantie et } \\
\text { qualifiée }\end{array}$ & $\begin{array}{l}\text { Assertion comportant l'apport de garanties qui la justifient et la } \\
\text { délimitation de sa validité }\end{array}$ & $\begin{array}{l}\text { "Uh petit apport venant de la méthode de ma tutrice (valable pour le collège) : } \\
\text { au début de lannée, les élèves signent un contrat de travail qui indique quils } \\
\text { apprendront pour chaque cours la leçon précédente... Les intemagations ne sont } \\
\text { jamais annoncées. Ses élèves le savent. Apparemment ça fonctionne a peu près.» }\end{array}$ \\
\hline $\begin{array}{l}\text { En dehors de la } \\
\text { tâche }\end{array}$ & Questions, digressions ou tout énoncé sans rapport avec le débat & $\begin{array}{l}\text { "Alors, à ceux qui estiment que ma contribution parasite le forum, Mea Culpa, } \\
\text { et je m'éclipse... \$ }\end{array}$ \\
\hline
\end{tabular}

Tableau 4 : Extrait de la table des règles pour le codage des segments (dimension argumentative du forum débat IES)

A noter que la méthode des juges est aussi utilisée pour le codage des segments dans chacune des dimensions étudiées : notre analyse finale du corpus résulte bien du croisement de ces analyses différenciées des dimensions argumentative, épistémique et sociale.

\subsection{Analyse qualitative des segments par catégorie}

L'analyse qualitative des segments dans chacune des dimensions permet de donner des interprétations fines des catégories les plus représentatives quantitativement. Ainsi dans le forum débat IES nous avons pu montré la centration du débat sur le suivi de l'apprentissage des élèves avec la mobilisation d'un schème d'action assez vaste concernant le suivi régulier du travail des élèves dont l'IES devient une simple règle d'action.

La figure 3 propose, selon le modèle de Coulet, notre interprétation du contenu des 169 segments associés à ce schème et ses formes de régulation. Nous visualisons par cette modélisation une compétence en construction, très partagée et mobilisée par les professeurs stagiaires face au problème du suivi du travail des élèves en dehors de la classe ou du moins nous accédons à la verbalisation de cette compétence dans cet exercice collectif d'analyse de la pratique. 


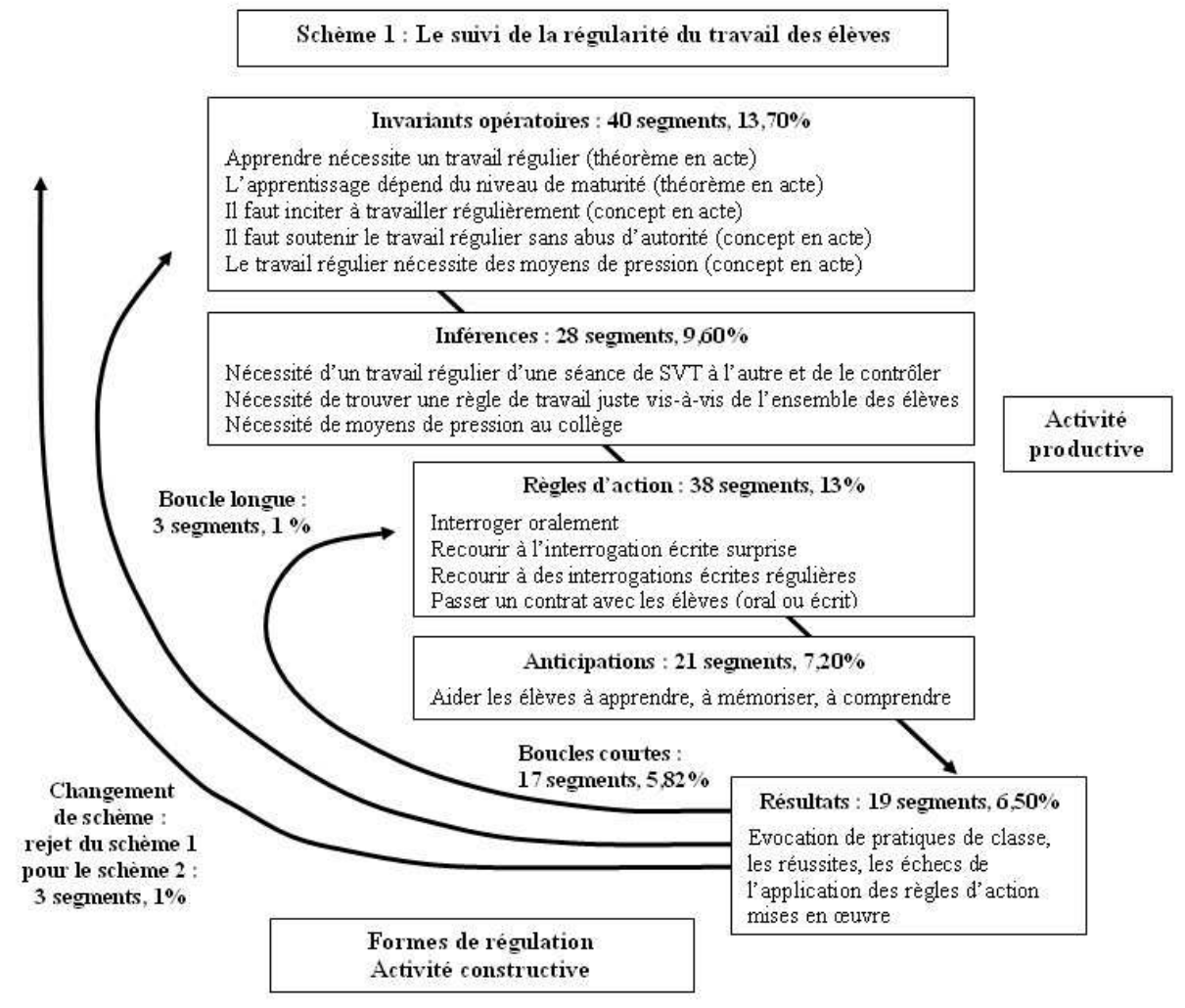

Figure 3 : Modélisation de la construction d'une compétence en rapport avec le suivi du travail des élèves (forum débat IES)

\section{Utilisation du cadre d'analyse - Quelques résultats appliqués au forum débat IES}

Il s'agit dans ce paragraphe de ne reprendre que quelques grandes lignes de notre démarche, le minimum indispensable pour que d'autres équipes de chercheurs l'appliquent à d'autres corpus. Cela nous permettrait de procéder à une étude comparative qui nous renseignerait utilement sur la validité de notre démarche et probablement enrichirait notre questionnement. Le lecteur qui souhaite approfondir certains points et prendre connaissance de manière plus approfondie des résultats qu'elle a produits pourra se reporter aux articles que nous avons régulièrement produits (voir bibliographie).

Les premières données issues de l'approche quantitative du corpus permettent de comparer les segmentations dans les trois dimensions, de voir si les grains de segmentation sont comparables ou pas et de quantifier également ce qui est hors propos vis-à-vis des analyses qui seront conduites ensuite. Le tableau 5 propose les résultats de nos différentes segmentations des 54 messages du forum débat IES de 2004-2005 postés par 30 professeurs stagiaires de SVT (82\% de participation) et 3 formateurs.

\begin{tabular}{|l|c|c|c|c|c|c|}
\hline & \multicolumn{2}{|c|}{$\begin{array}{c}\text { Dimension } \\
\text { épistémique }\end{array}$} & \multicolumn{2}{c|}{$\begin{array}{c}\text { Dimension } \\
\text { argumentative }\end{array}$} & \multicolumn{2}{c|}{$\begin{array}{c}\text { Dimension } \\
\text { sociale }\end{array}$} \\
\hline Nombre de segments & 433 & $100 \%$ & 403 & $100 \%$ & 475 & $100 \%$ \\
\hline Accord entre les deux codeurs & 433 & $100 \%$ & 390 & $96,8 \%$ & 464 & $97,7 \%$ \\
\hline Kappa de Cohen & \multicolumn{2}{|c|}{1} & \multicolumn{2}{|c|}{0,93} & \multicolumn{2}{c|}{0,94} \\
\hline Interactions hors tâche & 52 & $12 \%$ & 52 & $13,3 \%$ & 67 & $14,4 \%$ \\
\hline
\end{tabular}

Tableau 5 : Segmentation du corpus 
Deux à trois tours de segmentation ont généralement suffi pour établir un accord total ou presque des codeurs. Nous constatons que les segmentations sont comparables, que les unités de sens dégagés dans les trois dimensions ont à peu près le même grain.

Les résultats statistiques du tableau 5 indiquent qu'environ $12 \%$ à $14 \%$ seulement du corpus selon les dimensions s'écarte du sujet proposé. Il s'agit essentiellement de digressions humoristiques et d'un début de conflit entre trois professeurs stagiaires ayant rapidement fait l'objet d'une intervention du tuteur. L'engagement dans la tâche peut donc être considéré comme excellent, les conditions de l'acquisition d'un savoir par les professeurs stagiaires sont réunies (Cohen, 1994).

Les procédures utilisées dans les analyses des segments permettent de dégager des lignes d'interprétation dans chaque dimension que nous quantifions à chaque fois. La figure 3 proposée précédemment illustre la reconstitution d'une compétence collective en construction (dimension épistémique) dont chaque élément tiré d'une interprétation qualitative est quantifié de manière à donner à cette interprétation un certain poids, une certaine objectivité par rapport à notre grille d'analyse. Nous pouvons ainsi affirmé que cette interprétation des chercheurs ne se base pas sur un pourcentage minime du corpus qui serait mis ainsi en exergue mais résulte bien de l'analyse d'une part importante de celui-ci (169 segments sur les 381 analysés soit $44 \%$ du corpus).

La figure 4 propose une reconstitution de la trame argumentative du débat pris comme second exemple (dimension argumentative). On distingue deux rapports principaux à l'IES. Une majorité des intervenants se place dans une dynamique favorable à cette pratique (11 prises de positions), une minorité active produisant plutôt des prises de position défavorables (3 prises de position). Chacune de ses prises de position fait l'objet d'un nombre variables d'arguments (A), de contre arguments (C) et de répliques (R).

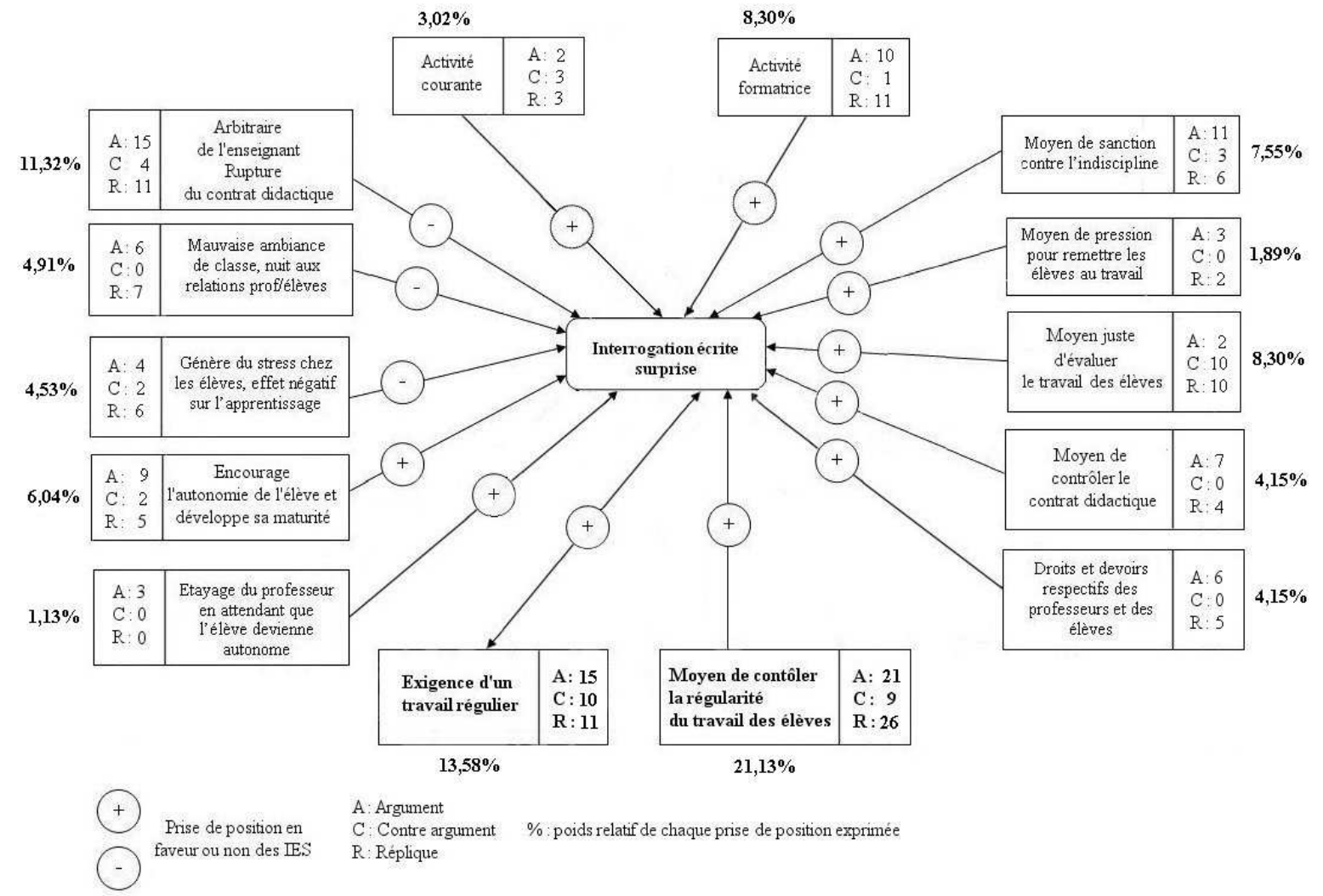

Figure 4 : Trame argumentative du débat IES 2004-2005 
Ce schéma explicite bien notre démarche. Toutes les prises de position n'ont pas la même valeur dans ce débat. On comprend alors facilement que sans la quantification qui les accompagne, on ne peut percevoir où se trouvent les positions majoritaires. Notre approche à la fois quantitative et qualitative des segments nous a permis de mettre en évidence dans notre exemple qu'une idée forte transcende l'opposition, et se trouve à la fois dans les messages favorables et défavorables à l'IES : celle de l'exigence d'un travail régulier de la part des élèves et de son contrôle (en gras sur la figure 4 : 32 messages, 92 segments, 36 arguments, 19 contre arguments et 37 répliques).

\section{Perspectives pour la formation professionnelle}

Nous pensons disposer d'un cadre d'analyse intéressant parce qu'il permet de dépasser cette première vue d'un forum comme une masse informe d'informations sans lien apparent les unes avec les autres - comme ce fut le cas lors de notre premier contact avec le corpus. Dans une préoccupation de mise des résultats de la recherche au service des praticiens, nous nous sommes questionnés pour trouver un bon compromis entre temps à investir dans une démarche de ce type et accès à ce potentiel pour l'intervention du formateur. La figure 5 présente un cadre d'analyse allégé (dimension épistémique) utilisé en formation, plus précisément en analyse de pratique.

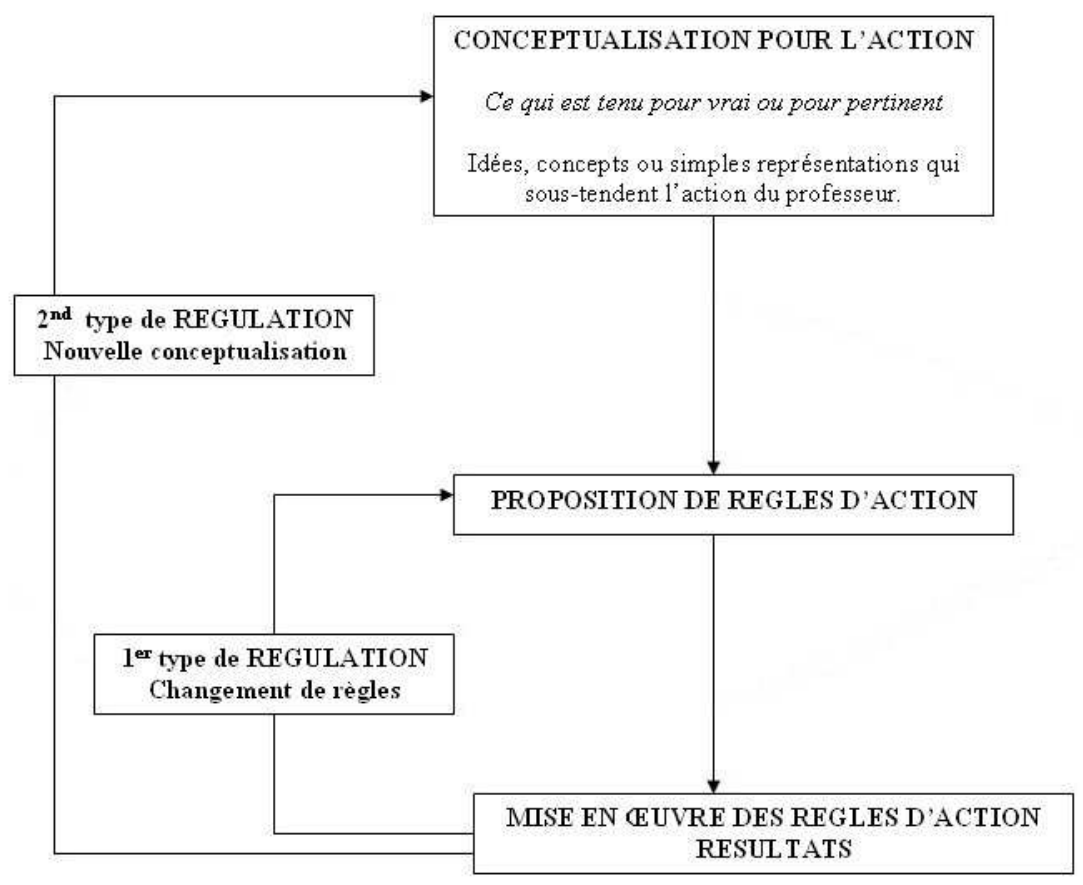

Figure 5 : Un outil didactique dérivé du modèle de construction de compétence de Coulet

Cet outil a été expérimenté sur plusieurs forums de discussion, dans diverses conditions et auprès de formateurs non spécialistes: analyse par un formateur disciplinaire (SVT) de quelques messages du forum IES présenté et analyse par un formateur en sciences humaines et sociales et par quelques professeurs stagiaires, dans une séance en présentiel, de quelques messages d'un forum d'analyse de pratiques consacré à l'autorité en classe (exemple du message de Céline, tableau 6). 


\begin{tabular}{|c|c|c|}
\hline $\begin{array}{c}\text { Message de Céline qui s'adresse à Sophie } \\
\text { Elle pensait instaurer le dialogue directement... En } \\
\text { revoyant ses représentations, elle a modifié ses règles } \\
\text { d'action... }\end{array}$ & $\begin{array}{l}\text { Interprétation par un } \\
\text { formateur en SHS }\end{array}$ & $\begin{array}{l}\text { Interprétation par des } \\
\text { professeurs stagiaires }\end{array}$ \\
\hline $\begin{array}{l}\text { Je prends quelques minutes pour mettre les pieds dans le } \\
\text { plat. Il me semble que tu confonds toi-même respect du } \\
\text { prof pour ses élèves et faiblesse. J'ai confondu aussi. A } \\
\text { Roubaix, avec une classe de cinquième. Il m’a semblé à } \\
\text { ce moment qu'en étant à l'écoute de mes élèves, en leur } \\
\text { montrant que j'acceptais le dialogue avec eux, je leur } \\
\text { prouverais qu'ils pouvaient me faire confiance et que } \\
\text { tout irait bien. Je me suis trompée. J'en ai bavé. J'ai failli } \\
\text { démissionner. Je pense maintenant que la confiance, ça } \\
\text { se gagne. Je pense être ce qu'on appelle une prof } \\
\text { autoritaire. Je gronde, je fais les gros yeux, au début de } \\
\text { l'année, il m'arrive même de punir ou de coller. Mais je } \\
\text { ne renvoie pas les élèves, je n'ai fait aucun rapport au } \\
\text { principal cette année. Je ne suis pas magique, ni } \\
\text { envoûtée. Mon "autorité" n'est pas naturelle, je me la } \\
\text { suis construite. Si j'essaie de m'imposer à mes élèves, de } \\
\text { me montrer autoritaire, de leur montrer que c'est moi qui } \\
\text { commande, en un mot, si je suis sévère, c'est par respect } \\
\text { pour eux. Demander à des gamins de } 12 \text { ou } 13 \text { ans de } \\
\text { savoir ce qui est bon pour eux, de savoir quand s'arrêter, } \\
\text { de connaître les limites sans aucun rappel, sans } \\
\text { apprentissage, c'est ce qui me semble être un manque de } \\
\text { respect. [...] Tu dis qu'on confond souvent respect et } \\
\text { faiblesse? Moi, je dis qu'on confond également autorité } \\
\text { et abus de pouvoir. On peut exiger de travailler dans le } \\
\text { calme, on peut demander aux élèves de lever la main } \\
\text { pour que tout le monde soit entendu, sans hurler, faire } \\
\text { une tête de bouledogue, punir à tours de bras, coller, } \\
\text { exclure, casser... Il faut juste trouver son créneau. Moi, } \\
\text { c'est un bon vieux système de point d'oral à l'ancienne } \\
\text { et un humour assez décapant, jamais méchant. Mais il a } \\
\text { surtout fallu me convaincre moi-même que l'étape du } \\
\text { "c'est moi le chef" était non seulement nécessaire à tout } \\
\text { embryon de dialogue, mais encore une étape attendue par } \\
\text { les élèves. [...] }\end{array}$ & $\begin{array}{l}\text { Conception: L'autorité est } \\
\text { naturelle. Il suffit d'être à } \\
\text { l'écoute des élèves, d'accepter } \\
\text { le dialogue } \\
\text { Résultat : échec } \\
\text { Régulation en boucle longue: } \\
\text { l'autorité n'est pas naturelle, } \\
\text { elle se construit } \\
\text { Règles : sévérité en début } \\
\text { d'année, ne rien laisser passer, } \\
\text { punir, coller } \\
\text { Les représentations actuelles } \\
\text { de Céline qui conditionnent } \\
\text { son action en classe: } \\
\text { - avoir du respect pour les } \\
\text { élèves, c'est leur apprendre les } \\
\text { règles à ne pas dépasser. } \\
\text { - les élèves ne sont pas assez } \\
\text { mûrs pour savoir ce qui est } \\
\text { bon pour eux. Ils ne } \\
\text { connaissent pas leurs limites } \\
\text { sans aucun rappel, sans } \\
\text { apprentissage. } \\
\text { - ne pas confondre autorité et } \\
\text { abus de pouvoir } \\
\text { - la sévérité est nécessaire, elle } \\
\text { est attendue par les élèves } \\
\text { avant tout dialogue }\end{array}$ & $\begin{array}{l}\text { Conception : même idée } \\
\text { que Sophie sur l'autorité } \\
\text { naturelle du professeur, } \\
\text { liée en particulier à son } \\
\text { statut. } \\
\text { Elle s'est trompée et elle } \\
\text { pense différemment } \\
\text { maintenant (régulation) } \\
\text { Des actions : je gronde, } \\
\text { je fais des gros yeux, je } \\
\text { colle, etc.. } \\
\begin{array}{l}\text { Des résultats : le } \\
\text { dialogue s'instaure } \\
\text { progressivement, les } \\
\text { élèves font confiance } \\
\text { Les idées qui sous- } \\
\text { tendent son action: } \\
\text { - les élèves ne } \\
\text { connaissent pas leurs } \\
\text { limites, la sévérité est } \\
\text { nécessaire avant tout } \\
\text { dialogue } \\
\text { - il ne faut pas confondre } \\
\text { autorité et abus de } \\
\text { pouvoir } \\
\text { - le professeur doit rester } \\
\text { le chef mais cette } \\
\text { autorité se construit } \\
\text { progressivement }\end{array}\end{array}$ \\
\hline
\end{tabular}

Tableau 6 : Un court extrait du forum «Exercer l'autorité dans la classe : qu'en pensent de jeunes enseignants?»-IUFM de Lille - 2005 http://www.lille.iufm.fr/passages/article.php3?id\%20article $=281$

Cette première expérimentation de l'usage en formation du cadre d'analyse créé pour des besoins de recherche nous paraît bien concluante. Ce qui a été réalisé à partir d'un cadre d'analyse allégé nous encourage à persister dans cette direction et à investir dans la recherche d'une possible automatisation de certaines étapes de notre démarche méthodologique. Un travail est amorcé dans cette perspective au sein de l'ERTé CALICO (Conception pour l'apprentissage en ligne, Instrumentation, Collaboration) dans laquelle s'inscrit notre groupe de recherche.

\section{Conclusion}

En déployant notre méthodologie d'analyse dans cet article, nous espérons avoir apporter une double contribution, à la fois à la communauté des chercheurs et à la fois aux praticiens. 
Notre recherche illustre tout l'intérêt d'une approche intégrée (l'analyse qualitative et quantitative se complétant mutuellement) ainsi que d'une analyse en finesse et dans la durée pour éclairer la complexité du processus de construction de connaissances au sein d'un forum en ligne. Il illustre également et questionne la pertinence de détourner des outils méthodologiques pour un usage autre que celui pour lesquels ils ont été conçus (utilisation de la grille de Weinberger \& Fischer pensée pour des étude de cas, et enrichissement de la dimension épistémique par l'usage du modèle de Coulet inspiré de Vergnaud).

Du côté de la pratique, l'expérimentation que nous avons faite d'un usage de ces mêmes outils pour la pratique révèle un potentiel prometteur pour l'action et tout particulièrement pour l'intervention des formateurs accompagnateurs et tuteurs des forums. Deux choses nous restent cependant à affiner pour opérationnaliser à plus large échelle: revenir sur la simplification et l'automatisation des activités que suppose notre modèle ; appliquer notre modèle d'analyse à d'autres corpus dans la perspective d'une généralisation de cet outil créé dans le cadre de la recherche et proposé pour la pratique.

\section{Bibliographie}

AUDRAN, J. \& DAELE, A. Forums et liste de diffusion : rapport à la communauté et microculture, 2006

BAKER, M.J., HANSEN, T., JoIner, R. \& TRAUM, D. The role of grounding in collaborative learning tasks. In P. Dillenbourg (Ed.), Collaborative Learning : Cognitive and Computational Approaches, pp. 31-63. Amsterdam : Pergamon / Elsevier Science, 1999

CHI M. Th. (1997) "Quantifying qualitative analysis of verbal data: a practical guide", The journal of the learning sciences $n^{\circ} 6(3)$, p. 271-315.

COHEN J. (1960) «A coefficient of agreement for nominal scales », Educ. Psychol. Meas., $\mathrm{n}^{\circ} 20$, p. $27-46$

Coulet J.C \& Chauvigne C. (2005) «Passer d'un référentiel de compétences à une ingénierie de formation »Education Permanente, n¹65, p.101-113

CIUSSI, M. Dynamique des liens sociaux à distance : genèse des formes et processus observables, colloque AREF, Strasbourg

DESJARDIN C. (2002). Vers une méthodologie d'analyse des construits cognitifs collaboratifs produits dans les forums électroniques textuels asynchrones. - Thèse - Université de Montréal

Dillenbourg P., BAKer M., Blaye A, O'MAlley C., (1996) « The evolution of research on collaborative learning » In E. Spada \& P. Reiman (Eds) Learning in Humans and Machine : Towards an interdisciplinary learning science , Oxford : Elsevier, 1996, p. 189-211.

De WeVer, B., Schellens, T., Valcke, M., Van Keer, H. (2006) Content analysis schemes to analyze transcripts of online asynchronous discussion groups : a review, Computers and education, 46, 6-28

JOHNSON \& JOHNSON (1999) Learning together and alone : cooperative, competitive and individualistice leazrning. Boston : Allyn \& Bacon, 1999

KuHn D. (1991) The skills of argument. New-York : Cambridge University Press

KUSTER Y.\& LAMEUL G. (2008). «Le forum débat argumentatif : un outil de formation et d'analyse de pratique », in Sidir M. (coord) Usages des outils et médias éducatifs, traité Sciences et techniques de l'Information, Paris : Hermes. 
KUSTER Y \& LAMEUL G. (2008) «Le forum débat, un espace de construction de compétences professionnelles en formation initiale des enseignants », in Actes du colloque JOCAIR, 2729/08/2008, p.43-55

LAMEUL G. (2006] Former des enseignants à distance ? Etude des effets de la médiatisation de la relation pédagogique sur la construction des postures professionnelles enseignantes, Thèse soutenue à l'université de Paris X - Nanterre, 2006.

LAMEUL G. et KuSTER Y. (2009). Analyse de la dimension socio-collaborative d'un débat sur forum, in J. Audran (coord) "Communautés d'apprenants en ligne, apprentissage et socialisation » Education et Formation.

LEITÄO S. (2000) «The potential of argument in knowledge building » Human Development, $\mathrm{n}^{\circ} 43$, p. 332-360.

Sensevy G., Kuster Y., Helary F, \& LAmeul G.,( 2005) Le forum débat : un dispositif d'apprentissage collaboratif en formation initiale d'enseignants, in Distances et Savoirs 3.

TEASLEY S. (1997). Talking about reasoning : how important is the peer in peer collaboration? In L.B. Resnick, R. Saljö, C. Pontecorvo \& B. Burges (Eds) Discourse tools and reasoning : Essays on situated cognition, Berlin : Springer, p. 361-384

TOULMin S.E (1958, 1993 en français) Les usages de l'argumentation. Paris : PUF.

VERGNAUD G. (1990) «La théorie des champs conceptuels », Recherche en didactique des mathématiques $\mathrm{n}^{\circ} 102 / 3$, p.133-170

WEBB, N.M. (1989) Peer interaction and learning in small groups, in International Journal of Educational Research, 13, 1989, p. 21-29

WEINBERGER A, FISHER F. (2006) «A framework to analyse argumentative knowledge construction in computer-supported collaborative learning ». Computers \& education $\mathrm{n}^{\circ} 46 \mathrm{p}$. 71-95.

\section{Courriels des auteurs}

yves.kuster@bretagne.iufm.fr

geneviève.lameul@bretagne.iufm.fr 